\title{
Diet-Dependent Immunohistochemical Evaluation of Connexin 43 in the Sheep Rumen
}

\author{
Rasha S. Ahmed ${ }^{1}$, Holger Martens ${ }^{2}$, Christoph Muelling ${ }^{3}$ \\ ${ }^{1}$ Department of Anatomy, Faculty of Veterinary Medicine, University of Khartoum, Khartoum, Sudan \\ ${ }^{2}$ Institut of Physiology, Faculty of Veterinary Medicine (WE02), Berlin, Germany \\ ${ }^{3}$ Institut of Anatomy, Faculty of Veterinary Medicine, Leipzig, Germany
}

Email address:

umraghd72@yahoo.com (R. S. Ahmed), holger.martens@fu-berlin.de (H. Martens), c.muelling@vetmed.uni-leipzig.de (C. Muelling)

\section{To cite this article:}

Rasha S. Ahmed, Holger Martens, Christoph Muelling. Diet-Dependent Immunohistochemical Evaluation of Connexin 43 in the Sheep Rumen. Animal and Veterinary Sciences. Vol. 4, No. 1, 2016, pp. 11-14. doi: 10.11648/j.avs.20160401.13

\begin{abstract}
The objective of this study was to characterize the immunohistochemical localization of plasma membrane connexin 43 in the rumen of sheep after changing the diet from hay (ad libitum) to a mixed hay/concentrate diet. A total of 24 sheep were fed mixed hay/concentrate for different periods ranging from 0 weeks (control; hay ad libitum) to 12 weeks (1-1.5 $\mathrm{kg}$ hay plus $780 \mathrm{~g}$ concentrate per day in two equal portions). Using immunohistochemical technique the present study confirmed the existence of plasma membrane connexin 43 in the sheep rumen epithelium. Plasma membrane connexin 43 immunostaining was most intense at the stratum basale and stratum spinosum (suprabasal layer) and decreased iron intensity through stratum spinosum (superficial layers) to stratum granulosum. Meanwhile, stratum corneum was negative. The reaction around the cells gave a syncitial appearance with more apical-immunostaining concentration. Moreover, the present study confirmed a significant effect of concentrate diet on the immunoreactivity of plasma membrane connexin 43 in the rumen of sheep. A very strong degree of antibody reaction was seen in 4 to 12 weeks concentrate-fed groups.
\end{abstract}

Keywords: Sheep, Rumen, Connexin 43, Feed, Immunohistochemistry

\section{Introduction}

The members of the connexin family are integral membrane proteins that forms hexamers called connexons. Most cells express two or more connexins. Gap junction channels are formed by docking of two connexons and are found at cell-cell appositions. Gap junction channels are responsible for direct intercellular transfer of ions and small molecules including propagation of inositol trisphosphatedependent calcium waves (Saez et al., 2003). In transmission electron micrographs of ultrathin tissue sections, gap junctions appear as regions where the plasma membranes of adjacent cells closely approach each other, but appear to be separated by a small gap of 2-3 nm (Benedetti and Emmelot, 1965, Revel and Karnovsky, 1967). Connexin 43 is the most ubiquitously expressed vertebrate gap -junction protein (Saez et al., 2003). Gap junctions, connexins, and functional intercellular coupling have been identified in multiple portions of the mammalian gastrointestinal tract. Along the gastrointestinal tract, connexin 26 and Connexin 32 are found in many of the epithelial cells, whereas connexin 43 is found in some epithelial cells but in most of the smooth muscle cells (Saez et al., 2003). In human skin, connexin 43 is found to be the predominant gap junction protein expressed in the stratum granulosum and stratum spinosum; however, it is minimal in stratum basale or absent in the stratum corneum (Salomon et al., 1994). In human skin, immunoelectron microscopy has localized connexin 43 to close membrane apposition (e.g., pentalaminar junctions adjacent to desmosomes) and has shown that the cellular distribution mapped by immunofluorescence matched that immunoelectron microscopy (Tada and Hashimoto, 1997). All gap junctions contain the same proteins; however, they differ in their electrophoretic mobilities (21-70 KDa) of band detected by SDS-PAGE in gap junction-enriched preparation of different tissues (Henderson et al., 1979, Hertzberg and Gilula, 1979, Kistler et al., 1985). In bovine, western analysis confirmed expression of connexin 43 with a single band of $\sim 43 \mathrm{KDa}$ in the rumen epithelium. Plasma membrane connexin 43 immunostaining is most intense at the stratum granulosum and decreases in intensity throughout stratum spinosum and stratum basale. Meanwhile, the stratum corneum is negative. Pattern of staining is consistent with intercellular communication within and between the cells of the stratum granulosum, spinosum and basale, forming a syncytium (Graham and Simmons, 2005). Connexin 26 immunofluorescence was 
present in cells of the stratum granulosum and stratum spinosum, whereas the stratum corneum and stratum basale were negative (Graham and Simmons, 2005).

\section{Materials and Methods}

\subsection{Experimental Animal}

A total of 24 of German dairy sheep of different sex were used in this study. Animals were 9-10 months old at the time of the experiment, and their weights ranged between 33.5-50 $\mathrm{kg}$. The animals were divided into 8 groups of three animals in each group.

\subsection{Feedings}

Prior to the experiment the sheep were fed only hay ad libitum for at least 8 weeks, in order to adapt them to a lowenergy food. Thereafter, the sheep were either solely fed hay (1-1.5 kg hay/day) (control-group) or received, in addition to that, $780 \mathrm{~g}$ concentrate (experimental-groups). The experimental animals were assigned to 7 groups according to the duration of concentrate feeding: 2, 4 days, 1, 2, 4, 6 and 12 weeks. Introduction of concentrate diet was preceded by an adaptation period of 4 days in which the concentrate feed was increased gradually. The concentrate diet was supplied in equal portions at $07.00 \mathrm{am}$ and $02.30 \mathrm{pm}$. All animals had free access to tap water and salt block.

\subsection{Compositions of Diet}

The nutrient content of both concentrate and hay rations were shown in tables 1 and 2 , respectively.

Table 1. Composition of the concentrate diet.

\begin{tabular}{llll}
\hline Nutrients & $\mathbf{\%}$ & Nutrients & $\%$ \\
\hline Dry matter & 89.1 & Organic ADF & 13.28 \\
Crude ash & 6.42 & Organic NDF & 25.46 \\
Crude protein & 18.03 & ADL & 3.99 \\
Crude fiber & 9.65 & Vitamin A & $7200 \mathrm{Iu}$ \\
Calcium & 0.65 & Vitamin D3 & $1800 \mathrm{Iu}$ \\
Phosphorus & 0.59 & Selenium & $0.5 \mathrm{mg}$ \\
Magnesium & 0.27 & Copper & $10 \mathrm{mg}$ \\
Potassium & 1.35 & DCAB & $+299 \mathrm{meq} / \mathrm{kg} \mathrm{DM}$ \\
Sodium & 0.42 & Net energy lactation (NEL) & $6.7 \mathrm{MJ} / \mathrm{kg}$ \\
Chloride & 0.46 & Metabolic energy (ME) & $10.41 \mathrm{MJ} / \mathrm{kg}$ \\
Sulfur & 0.21 & & \\
\hline
\end{tabular}

Table 2. Composition of hay diet.

\begin{tabular}{llll}
\hline Nutrients & $\mathbf{\%}$ & Nutrients & \% \\
\hline Dry matter & 93.5 & ADL & 4 \\
Crude ash & 4.9 & Non fibers carbohydrates (NFC) & 22.5 \\
Crude protein & 8.8 & Metabolizable energy (ME) & $9.3 \mathrm{MJ} / \mathrm{Kg}$ \\
Crude fiber & 29.3 & Net energy lactation (NEL) & $5.5 \mathrm{MJ} / \mathrm{Kg}$ \\
Potassium & 1.44 & Usable crude protein (nXP) & $120.3 \mathrm{~g} / \mathrm{Kg}$ \\
Sodium & 0.032 & Degradable crude protein (UDP) & $17.6 \mathrm{~g} / \mathrm{Kg}$ \\
Organic ADF & 34 & Ruminal nitrogen balance (RNB) & $-5.2 \mathrm{~g} / \mathrm{Kg}$ \\
Organic NDF & 56.5 & & \\
\hline
\end{tabular}

\subsection{Tissue Collection and Preparation}

Following the feeding trial and at the end of each experimental period, Full thickness mucosa or rumen papillae isolated directly with scissors from identical site of the rumen (left wall of the recessus ruminis ventral sac; adjacent to the left longitudinal groove) (Gäbel et al, 1987). Immunohistochemical staining was performed on both cryostat sections and on deparaffinized sections of the ruminal epithelium. Full thickness mucosa or rumen papillae were chilled with liquid nitrogen, and stored at $-76{ }^{\circ} \mathrm{C}$. Cryostat sections were cut $5 \mu \mathrm{m}$ thick and collected on microscope slides precoated with Silane solution (3Aminopropyl) (Co. Sigma, Code No. A3648). The sections were fixed in acetone for 9 sec. and dried for at least $1 \mathrm{~h}$ at room temperature, then refrozen and stored at $-20{ }^{\circ} \mathrm{C}$. Prior to staining the sections were dried $1 \mathrm{~h}$ at room temperature, and fixed in cold aceton for $8 \mathrm{~min}$. The ruminal mucosa was cleaned by immersion in a transportation buffer solution (see table 3). Fresh rumen- mucosal tissues were fixed in $4 \%$ formalin solution for 24 hours (Romeis, 1989). After rinsing with water, samples were stored in $0.1 \mathrm{mmol} / \mathrm{L}$ phosphate buffer ( $\mathrm{pH}$ 7.2). They were then dehydrated in graded series of ethanol alcohol, cleared with xyline, saturated with and embedded in paraffin. Tissue blocks were then cut at 5-7 $\mu \mathrm{m}$ thickness and collected on microscope slides precoated with Silane solution. Tissues were deparaffinized, hydrated, rinsed twice with distilled water before immunostaining.

Table 3. Composition of the transport buffer.

\begin{tabular}{ll}
\hline Compound & Concentration $(\mathbf{m m o l} / \mathbf{L})$ \\
\hline Sodium & 145.2 \\
Potassium & 5 \\
Calcium & 1 \\
Magnesium & 1 \\
Bicarbonate & 25 \\
Chloride & 120 \\
Dihydrogenphosphate & 0.4 \\
Hydrogenphosphate & 2.4 \\
Glucose & 5 \\
pH value & 7.4 \\
Osmolarity & $300 \mathrm{mosmol} / \mathrm{L}$ \\
\hline
\end{tabular}

\subsection{Antibodies}

\subsubsection{Primary Antibody}

Mouse anti- Connexin 43 (Cx43) monoclonal antibody (Fa.Chemicon, USA and Canada) Catalog Nr.MAB3067.

\subsubsection{Secondary Antibody}

Peroxidase labelled polymer conjugated to goat anti-mouse immunoglobulins in Tris-HCl buffer containing carrier protein and an anti-microbial agent, Code N0. K4007 (FaDakoCytomation, Carpinteria, USA)

\subsubsection{Negative Controls}

The specificity of the staining reaction was determined in control experiments. These comprised either substitution of the primary antibody by PBS, normal mouse serum 1:200 (negative control Ig GI Cat, No. X0931, FaDakoCytomation, Carpinteria, USA), or omission of the primary antibody; in all cases, sections were negative.

\subsection{Procedure of Immunohistochemistry}

For immunolabeling of gap junction-Connexin 43 (Cx43), frozen and deparaffinized sections were stained according to the protocol DakoEnvision+System, Peroxidase (DAB) (FaDakoCytomation, Carpinteria / USA).Cx43 was detected 
in cryostat sections. All immunohistochemistry was repeated for material obtained three animals and examined directly under a light microscope (Leica DM LB, Leica Microsystems, Wetzlar, Germany).

Tissues were immersed in cold acetone at $4^{\circ} \mathrm{C}$ for $8 \mathrm{~min}$. Then they rinsed twice with distilled water for $5 \mathrm{~min}$ each, washed twice in PBS (PBS-Puffer to Dulbecco Cat. No.47302, Fa.Serva, Heidelberg, Deutschland) for $5 \mathrm{~min}$ each. Sections were subjected to inactivation of endogenous peroxidases by incubation at room temperature in $0.03 \%$ hydrogen peroxide containing sodium azide for 5 minutes. Then, the sections were rinsed twice with distilled water for 3 min each, washed in PBS for $10 \mathrm{~min}$. The sections were incubated either with the primary antibody at dilutions of 1:200 in PBS buffer or with control serum at dilutions of $1: 200$ (control experiments) for $30 \mathrm{~min}$ in a humidified chamber. The sections were washed three times in PBS (twice for $3 \mathrm{~min}$ each and once for $10 \mathrm{~min}$ ). Then, sections were incubated with the secondary antibody for $35 \mathrm{~min}$ at room temperature. The sections were washed three times in PBS (twice for $3 \mathrm{~min}$ each and once for $10 \mathrm{~min}$ ), and color was developed by incubation with $\mathrm{DAB}+$ Chromogensubstrate solution ( $1 \mathrm{ml}$ of buffered substrate solution, $\mathrm{pH} 7.5$, containing hydrogen peroxide and a preservative + one drop of 3, 3-Diaminobenzidin Chromogen solution) for 5-10 min. Sections were rinsed twice with ice cold- distilled water for 5 min each. Then, sections were counterstained with Mayer's Haematoxylin for $5 \mathrm{~min}$, rinsed in distilled water and washed with running tap water for $5 \mathrm{~min}$. Then sections were cleared three times in xyline for $5 \mathrm{~min}$ each, and mounted with Canada balsam permanent mounting media (Sigma-Aldrich).

\section{Results}

Using immunohistochemical technique the present study confirmed the existence of plasma membrane connexin 43 in the sheep rumen epithelium. Cell borders of the ruminal epithelium have different affinity to connexin 43 immunostaining (Cx43). Immunostaining for connexin 43 was found peripherally around the cells and was stronger in the apical membranes. Plasma membrane connexin 43 immunostaining was most intense at the stratum basale and the stratum spinosum (suprabasal layer), decreased iron intensity throughout stratum spinosum (superficial layers) to stratum granulosum. Stratum corneum was negative (Fig.1). The reaction around the cells gave a syncitial appearance with more apical-immunostaining concentration (fig. 1).
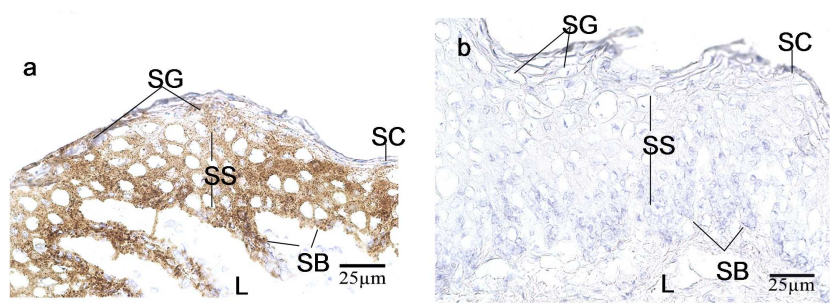

Fig. 1. Micrographs of the ruminal epithelium and lamina propria (L)a. Peripheral localization of connexin 43 in the cells of stratum basale (SB)and deep layers of stratum spinosum (SS)with decreasing intensity through the superficial spiny cells and stratum granulosum (SG). Stratum corneum was negative $b$. Negative control. (Scale $25 \mu \mathrm{m}$ )
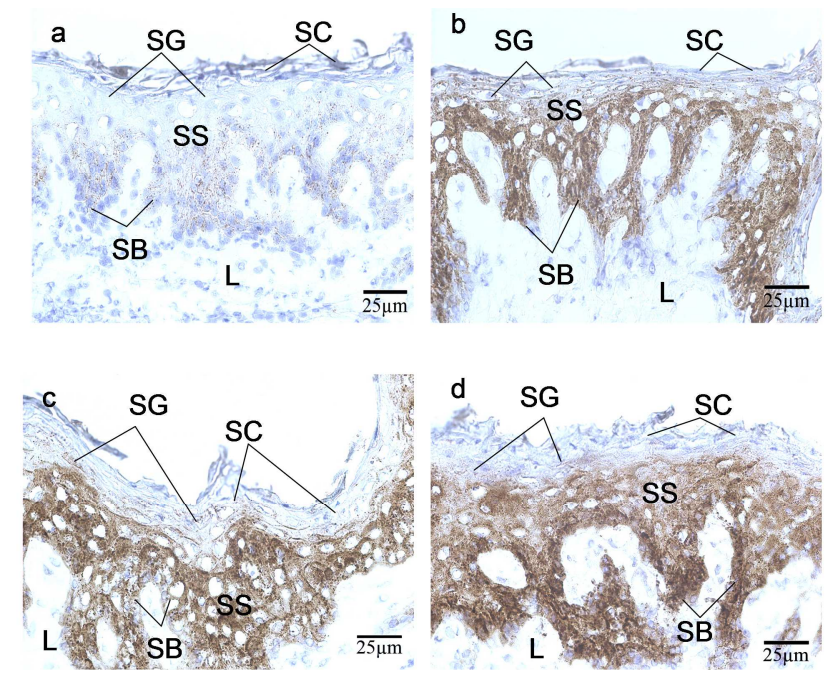

Fig. 2. Micrographs of the ruminal epithelium showing different immunoreactivity for connexin 43 among sheep groups: a. very weakreaction (hay-fed group) b. moderate reaction (one week concentrate-fed group) c. strong reaction (2 weeks concentrate-fed group) d. very strong reaction (4 weeks concentrate-fed group). (Scale 25 $\mu \mathrm{m}$ )

Table 4. The effect of type and duration of concentrate feeding on the expression of Cx43 at different strata of the rumen epithelium. $(N=3$ animals for each treatment).

\begin{tabular}{lllll}
\hline Animals & $\begin{array}{l}\text { Str. } \\
\text { granulosum }\end{array}$ & $\begin{array}{l}\text { Str. spinosum } \\
\text { (superficial) }\end{array}$ & $\begin{array}{l}\text { Str. spinosum (deep } \\
\text { or suprabasal } \\
\text { layer) }\end{array}$ & $\begin{array}{l}\text { Str. } \\
\text { Basale }\end{array}$ \\
\hline CF0W & + & ++ & ++ & ++ \\
CF2 D & + & ++ & ++ & ++ \\
CF4 D & + & ++ & +++ & +++ \\
CF1W & + & ++ & +++ & +++ \\
CF2W & ++ & +++ & ++++ & ++++ \\
CF4W & ++ & +++ & ++++ & +++++ \\
CF6W & ++ & +++ & ++++ & +++++ \\
CF12W & ++ & +++ & ++++ & +++++ \\
\hline
\end{tabular}

$+=$ very weak reaction $++=$ weak $+++=$ moderate $++++=$ strong $+++++=$ very strong

Moreover, the present study confirmed a significant effect of concentrate diet on the immunoreactivity of plasma membrane connexin 43 in the sheep rumen. The degree of the antibody-reaction differed between hay fed group and concentrate-fed groups (table. 4). Ruminal epithelium of hay fed group presented very weak to weak degree of reaction. However, the degree of the antibody-reaction within concentrate-fed groups was weak in 2 days concentrate-fed group, moderate in 4 days and 1 week concentrate-fed groups, strong in 2 weeks concentrate-fed group, and very strong in 4, 6 and 12 weeks concentrate-fed groups (Fig. 2; Table. 4).

\section{Discussion}

The present study showed that, plasma membrane connexin 43 immunostaining was most intense in the stratum basale and stratum spinosum (deep layers) and decreased in intensity through stratum spinosum (superficial layers) to stratum granulosum. Meanwhile, the stratum corneum was negative. The reaction around the cells gave a syncitial appearance with more apical-immunostaining concentration. The degree of antibody 
reaction was weak in hay-fed sheep and 2 days concentrate-fed sheep, moderate in 4 days and 1 week concentrate-fed sheep, strong in 2 weeks concentrate-fed sheep and very strong reaction in 4 to 12 weeks concentrate-fed sheep.

Gap junction communication mediated by the connexin gene family allows both cell-cell ionic coupling, that is, the intercellular passage of small ions such as potassium, and cellcell biochemical coupling facilitating the sharing of small molecules (metabolites, sugars, lactate, butyrate, etc.) or intercellular signaling molecules (cyclic nucleotides) (Saez, et al, 2003; White, 2003). The key concept of the arrangement for multicellular-stratified epithelia capable of active sodium transport (such as in the frog skin) is that the epithelial barrier layers communicate with lower basal cell layers via lowresistance intercellular gap-junctional pathways (Mills et al, 1977). Graham and Simmons (2005) showed that connexin-43 is immunolocalised to the stratum granulosum, stratum spinosum and stratum basale of the bovine rumen epithelium and is consistent with the formation of a functional syncytium between these cells. Furthermore, Shahin and Blankemeyer (1989) studied the morphology and distribution of the intercellular junctions in isolated skin of Ranapipiens using various electron-microscopic techniques. They demonstrated the presence of gap junctions and suggest that the distribution of gap junctions is not homogeneous among the epithelial strata. They are less frequent in the stratum corneum and stratum granulosum than in the stratum spinosum and stratum germinativum. The present findings are consistent with these finding; and a model of wide spread intercellular coupling, although the lower number of gap junctions in the stratum granulosum, suggested a possible deficiency in intercellular coupling. Gap junction channels are responsible for direct intercellular transfer of ions and small molecules including propagation of inositol trisphosphate-dependent calcium waves (Saez et al., 2003). Thus the increase in gap junctions, which were more pronounced in both stratum basale and deep stratum spinosum of 2-12 weeks concentrate-fed sheep, could be considered as adaptive mechanism for increasing the absorptive capacity of the rumen epithelium.

\section{Conclusion}

Using immunohistochemical technique the present study confirmed a significant effect on the immunoreactivity of plasma membrane connexin 43 in the rumen of sheep associated to the diet change. A very strong degree of antibody reaction was seen in 4 to 12 weeks concentrate-fed groups. Connexin 43 immunostaining was more intense at the stratum basale and stratum spinosum; stratum corneum was negative.

\section{Acknowledgements}

This research was funded by DAAD organization (Germany) and the University of Khartoum (Sudan).

\section{References}

[1] Benedetti, E. L. and Emmelot, P. (1965) Electron microscopic observations on negatively stained plasma membranes isolated from rat liver. J. Cell Biol. 26: 299-305.

[2] Gäbel, G.; Martens, H.; Suendermann, M. and Galfi, P. (1987) The effect of diet, intraruminal $\mathrm{pH}$ and osmolarity on sodium, chloride and magnesium absorption from the temporarily isolated and washed reticulo-rumen of sheep. Q. J. Exp. Physiol. 72: 501-511.

[3] Graham, C. and Simmons, N. L. (2005) Functional organization of the bovine rumen epithelium. Am. J. Physiol. Regul. Integr. Comp Physiol. 288: 173-181.

[4] Henderson, D.; Eibl, H. and Weber, K. (1979) Structure and biochemistry of mouse hepatic gap junctions. J. Mol. Biol. 132: $193-218$

[5] Hertzberg, E. L. and Gilula, N. B. (1979) Isolation and characterization of gap junctions from rat liver. J. Biol. Chem. 254: $2138-2147$

[6] Kistler, J.; Kirkland, B. and Bullivant, S. (1985) Identification of a 70,000-D protein in lens membrane junctional domains. J. Cell Biol. 101: 28-35.

[7] Mills, J. W.; Ernst, S. A. and DiBona, D. R. (1977) Localization of Na-pump sites in frog skin. J. Cell Biol. 73: 88-110.

[8] Revel, J. P. and Karnovsky, M. J. (1967) Hexagonal array of subunits in intercellular junctions of the mouse heart and liver. J. Cell Biol. 33: C7-C12.

[9] Romeis, B. (1989) Mikroskopische Technik.Verlag Urban und Schwarzenberg, München, Wien und Baltimore, 17. Auflage.

[10] Saez, J. C.; Berthoud, V. M.; Branes, M. C.; Martinez, A. G. and Beyer, E. C. (2003) Plasma membrane channels formed by connexins: Their regulation and functions. Physiol. Rev. 83: $1359-1400$.

[11] Salomon, D., Masgrau, E., Vischer, S., Ullrich, S., Dupont, E., Sappino, P., Saurat, J. H. and Meda, P. (1994) Topography of mammalian connexins in human skin. J. Invest. Dermatol. 103: $240-247$.

[12] Shahin, S. H. and Blankemeyer, J. T. (1989) Demonstration of gap junctions in frog skin epithelium. Am. J. Physiol. 257: C 658-664.

[13] Tada, J. and Hashimoto, K. (1997) Ultrastructural localization of gap junction protein connexin 43 in normal human skin, basal cell carcinoma and squamous cell carcinoma. J. Cutan. Pathol. 24: 628-635.

[14] White, T. W. (2003) Non redundant gap-junction functions. News Physiol. Sci. 18: 95-99. 